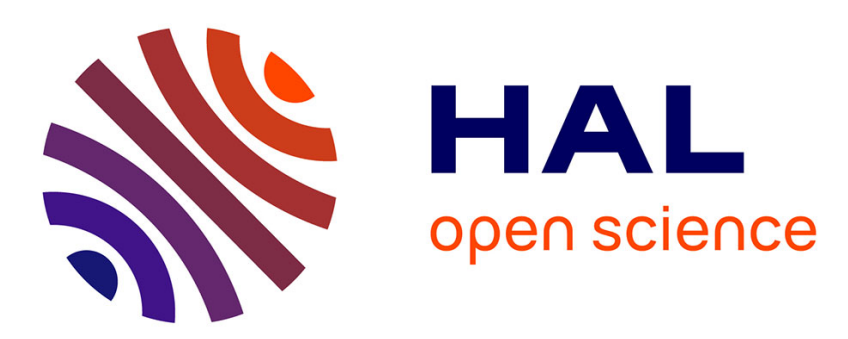

\title{
SemCaDo: a serendipitous strategy for learning causal bayesian networks using ontologies
}

\author{
Montassar Ben Messaoud, Philippe Leray, Nahla Ben Amor
}

\section{To cite this version:}

Montassar Ben Messaoud, Philippe Leray, Nahla Ben Amor. SemCaDo: a serendipitous strategy for learning causal bayesian networks using ontologies. The 11th European Conference on Symbolic and Quantitative Approaches to Reasoning with Uncertainty, Jun 2011, Belfast, Ireland. pp.182-193. hal-00596260

\section{HAL Id: hal-00596260 https://hal.science/hal-00596260}

Submitted on 27 Jun 2011

HAL is a multi-disciplinary open access archive for the deposit and dissemination of scientific research documents, whether they are published or not. The documents may come from teaching and research institutions in France or abroad, or from public or private research centers.
L'archive ouverte pluridisciplinaire HAL, est destinée au dépôt et à la diffusion de documents scientifiques de niveau recherche, publiés ou non, émanant des établissements d'enseignement et de recherche français ou étrangers, des laboratoires publics ou privés. 


\title{
SemCaDo: a serendipitous strategy for learning causal bayesian networks using ontologies
}

\author{
Montassar Ben Messaoud ${ }^{1,2}$, Philippe Leray ${ }^{2}$, and Nahla Ben Amor ${ }^{1}$ \\ 1 LARODEC, Institut Supérieur de Gestion Tunis \\ 41, Avenue de la liberté, 2000 Le Bardo, Tunisie. \\ benmessaoud.montassar@hotmail.fr, nahla.benamor@gmx.fr \\ ${ }^{2}$ Knowledge and Decision Team \\ Laboratoire d'Informatique de Nantes Atlantique (LINA) UMR 6241 \\ Ecole Polytechnique de l'Université de Nantes, France. \\ philippe.leray@univ-nantes.fr
}

\begin{abstract}
Learning Causal Bayesian Networks (CBNs) is a new line of research in the machine learning field. Within the existing works in this direction $[8,12,13]$, few of them have taken into account the gain that can be expected when integrating additional knowledge during the learning process. In this paper, we present a new serendipitous strategy for learning CBNs using prior knowledge extracted from ontologies. The integration of such domain's semantic information can be very useful to reveal new causal relations and provide the necessary knowledge to anticipate the optimal choice of experimentations. Our strategy also supports the evolving character of the semantic background by reusing the causal discoveries in order to enrich the domain ontologies.
\end{abstract}

\section{Introduction}

Bayesian networks (BNs), first introduced by Pearl [14], are compact graphical probabilistic models able to efficiently model uncertainty in real world problems.

One of the important properties relative to BNs is the Markov equivalence property which can be illustrated by the fact that the two networks $X \rightarrow Y$ and $X \leftarrow Y$ are equivalent (i.e. encode the same joint probability distribution). Nevertheless, only one of them is a correct from causal point of view. In fact, in the first network $X$ causes $Y$, then, manipulating the value of $\mathrm{X}$ affects the value of $\mathrm{Y}$ contrary to the second one where $Y$ is a cause of $X$ meaning that manipulating $X$ will not affect $Y$. Thus a BN cannot be considered as a proper causal network but the contrary is always true. This means that given a Causal Bayesian Network (CBN) one can use it even to determine how the observation of specific values (evidence) affects the probabilities of query variable(s) or to predict the effect of an intervention on the remaining variables [15].

Contrary to the non-Gaussian learning methods (also called LiNGAM) which use pure observational data $\left(D_{\text {obs }}\right)$, the causal discovery in CBNs often requires interventional data $\left(D_{\text {int }}\right)$. In this work, we don't make use of LiNGAM methods since no suitable parametrization of the joint distribution can be established 
when working under the non-gaussianity assumption. This is the key reason for restricting our approach to only CBNs.

This paper provides a substantially extended version of our previous work [1] in which we introduce the preliminary findings for integrating a semantic distance calculus to choose the appropriate interventions. Further developments along this direction have been made in order to deploy more efficient strategies to integrate the semantic prior knowledge, improve the causal discovery process and reuse the new discovered information.

The remainder of this paper is arranged as follows: Section 2 gives the necessary background for both CBNs and ontologies and discusses some related works that combine the two formalisms. Section 3 sets out how to use the ontological knowledge to enhance the causal discovery and vice versa. In Section 4, we show simulation results to evaluate the performances of the proposed algorithm. Concluding remarks and future works are given in Section 5.

\section{Basic concepts \& background}

\subsection{Causal Bayesian Networks}

A Causal Bayesian Network (CBN) is a Directed Acyclic Graph (DAG) where the set of nodes $\mathrm{V}$ represents discrete random variables $\mathrm{X}=\left\{X_{1}, X_{2}, . ., X_{n}\right\}$ and the set of edges E represents causal dependencies over V. We use $D_{i}$ to denote the finite domain associated with each variable $X_{i}$ and $x_{i}$ to denote any instance of $X_{i}$. We denote by $\operatorname{Pa}\left(X_{i}\right)$ the set of parents nodes for $X_{i}$ and $N e i\left(X_{i}\right)$ the set of its neighboring variables.

In addition to the usual conditional independence interpretation, the CBN is also given a causal interpretation since each directed edge is traduced as that the source node is the direct cause of the target node. For this reason, CBNs are considered as proper bayesian networks (BNs) but the reverse is not necessarily true.

The main difference between the two formalisms lies in the nature of the data needed to learn the structure. In fact, contrary to BNs, when using only observational data, we may not have enough information to discover the true structure of the graph and the causal model will be restricted to the Completed Partially Directed Acyclic Graph (CPDAG). Thus we have to collect further information on causality via interventions (i.e. actions tentatively adopted without being sure of the outcome). Here, we should note that intervening on a system may be very expensive, time-consuming or even impossible to perform. For this reason, the choice of variables to experiment on can be vital when the number of interventions is restricted.

All those distinguishing features have motivated many researchers to develop a variety of techniques and algorithms to learn such models $[8,12,13]$.

\subsection{Ontologies}

There are different definitions in the literature of what should be an ontology. The most notorious was given by Tom Gruber [7], stipulating that an ontology 
is an explicit specification of a conceptualization. The "conceptualization", here, refers to an abstract model of some phenomenon having real by identifying its relevant concepts. The word "explicit" means that all concepts used and the constraints on their use are explicitly defined.

In the simplest case, an ontology describes a hierarchy of concepts (i.e. classes) related by taxonomic relationships (is-a, part-of). In more sophisticated cases, an ontology describes domain classes, properties (or attributes) for each class, class instances (or individuals) and also the relationships that hold between class instances. It is also possible to add some logical axioms to constrain concept interpretation and express complex relationships between concepts.

Hence, more formally, an ontology can be defined as a set of labeled classes $\mathrm{C}=\left\{C_{1}, \ldots, C_{n}\right\}$, hierarchically ordered by the subclass relations (i.e. is-a, part-of relations). For each concept $C_{i}$ we identify k meaningful properties $p_{j}$, where $\mathrm{j} \in[1, \mathrm{k}]$. We use $H_{i}$ to denote the finite domain of instance (i.e. concretizing the ontology concepts by setting their properties values) candidates with each concept $C_{i}$ and $c_{i}$ to denote any instance of $C_{i}$. We also use $\mathrm{R}$ to represent the set of semantical (i.e non-hierarchical) relations between concepts and $R_{c}$ to represent the subset of causal ones. Finally, formal axioms or structural assertions $<c_{i}, c_{j}, s>$ can be included, where $s \in S$ is a constraint-relationship like "must, must not, should, should not, etc".

Practically speaking, the ontologies are often a very large and complex structure, requiring a great deal of effort and expertise to maintain and upgrade the existing knowledge. Such proposals can take several different forms such as a change in the domain, the diffusion of new discoveries or just an information received by some external source [6].

There are many ways to change the ontology in response to the fast-changing environment. One possible direction is the ontology evolution which consists in taking the ontology from one consistent state to another by updating (adding or modifying) the concepts, their properties and the associated relations [10].

The ontology evolution can be of two types [10]:

- Ontology population: When new concept instances are added, the ontology is said to be populated.

- Ontology enrichment: Which consists in updating (adding or modifying) concepts, properties and relations in a given ontology.

In order to establish the context in which the ontology evolution takes place, the principle of ontology continuity should be fulfilled [17]. It supposes that the ontology evolution should not make false an axiom that was previously true. When changes do not fulfill the requirement of ontological continuity, it is not any more an evolution, it is rather an ontology revolution.

\subsection{Related work}

Recent studies have investigated some ways to combine both ontologies and BNs. The first line of research focused on how to integrate the power of BNs to enhance 
the potential of ontologies by supplementing it with the principle means of modeling uncertainty in ontologies. In this way, [18] have proposed the OntoBayes approach, an ontology-driven Bayesian model for uncertain knowledge representation, to extend ontologies to probability-annotated OWL in decision making systems. [5] also proposed an approach when they augment the OWL language to allow additional probabilistic markups so that probability values can be attached to individual concepts and properties. One of the main advantage of this probabilistic-extended ontology is that it can support common ontology-related reasoning tasks as probabilistic inferences.

On the other hand, other solutions were proposed in order to enhance the $\mathrm{BN}$ construction by integrating ontologies. For example, [9] developed a semiautomatic BN construction system based on e-health ontologies. Their framework enables probabilistic inferencing capability for various E-health applications and contributes to reduce the complexity of BN construction. A similar approach for BN construction using ontologies was proposed in [4]. Nevertheless, it presents an automatic solution, implemented in the context of an adaptive, self-configuring network management system in the telecommunication domain.

To our knowledge, we are the first to propose a real cooperation in both ways between ontologies and CBNs. Our previous work [1] focused on only one facet of the $\mathrm{CBN}$-ontology combination by integrating the ontological knowledge to learn CBNs. Taking a further step in the same research direction, this work consists on designing a strategy that addresses issues to incorporate the second combination facet via reusing the causal discoveries to enrich the ontologies.

\section{SemCaDo: a serendipitous causal discovery algorithm for ontology evolution}

Generally, in the research area, scientific discoveries represent a payoff for years of well-planned works with clear objectives. This affirmation did not exclude the case of other important discoveries that are made while researchers were conducting their works in totally unrelated fields and the examples are abundant from Nobel's flash of inspiration while testing the effect of dynamite to Pasteur brainstorm when he accidentally discovered the role of attenuated microbes in immunization. In this way, we propose a new causal discovery algorithm which stimulates serendipitous discoveries when performing the experimentations using the following CBN-Ontology correspondences.

\subsection{CBNs vs Ontologies}

One of the main motivations when realizing this work is the similarities between CBNs and ontologies. This is particularly true when comparing the structure of the two models as shown in the following correspondences:

1. Nodes $\left(V_{i}\right) \leftrightarrow$ Concepts $\left(C_{i}\right)$ : The ontology concepts, which are relevant to the considered domain are traduced by the nodes of the CBN. 
2. Random variables $\left(X_{i}\right) \leftrightarrow$ Concept attributes $\left(C_{i} \cdot p_{j}\right)$ : All random variables in the CBN are represented as specific concept attributes in the ontology.

3. Causal dependencies $(\mathrm{E}) \leftrightarrow$ Semantic causal relations $\left(R_{c}\right)$ :

The correspondence between the two models in term of causality will be as follows:

- A causal relation between two concepts in the ontology will be traduced by a directed link between the corresponding CBN nodes. It is read as $c_{Y} \cdot p_{j}$ is the direct consequence of $c_{X} \cdot p_{j}$, where $p_{j}$ is the concept attribute used to make the correspondence.

- A causal dependency represented by a directed link in the CBN will be traduced by a specific causal relation between the appropriate concepts in the ontology.

4. Observational or experimental data $\left(D_{\text {obs }, i n t}\right) \leftrightarrow$ Concept-attribute instances $\left(c_{i} \cdot p_{j}\right)$ : We make a correspondence between the observational (resp. interventional) data at our disposal and the instances of the domain ontology. Each observation (resp. intervention) can be viewed, in the ontological context, as a state instantiation of a given concept attribute.

\subsection{SemCaDo Sketch}

Our approach relies on extending the MyCaDo algorithm [12] in order to incorporate available knowledge from domain ontologies. The original character of the SemCaDo (Semantic Causal Discovery) algorithm is essentially its ability to make impressive discoveries and reuse the capitalized knowledge in CBNs.

The correspondences between CBNs and ontologies in SemCaDo must respect the following constraints:

- Only a single ontology should be specified for each causal discovery task.

- Each causal graph node must be modeled by a corresponding concept in the domain ontology. The concepts which are candidates to be a member of such correspondence have to share the same studied attribute $p_{j}$.

- The causal discoveries concern concepts sharing the same semantic type (e.g. direct transcriptional regulation between genes). This means that all concepts $C_{i}$ modeled in the CBN must belong to the same super-concept $\mathrm{SC}$ and the causal relationship under study $R_{c}$ should be defined for any element of SC to any other one.

- The ontology evolution should be realized without introducing inconsistencies or admitting axiom violations.

In this way, we will adopt the same basic scenario as in MyCaDo and describe the possible interactions with the domain ontology.

The general overview of the SemCaDo algorithm is given in Figure 1. As inputs, SemCaDo needs an observational dataset and a corresponding domain ontology. Then it will proceed through three main phases: 


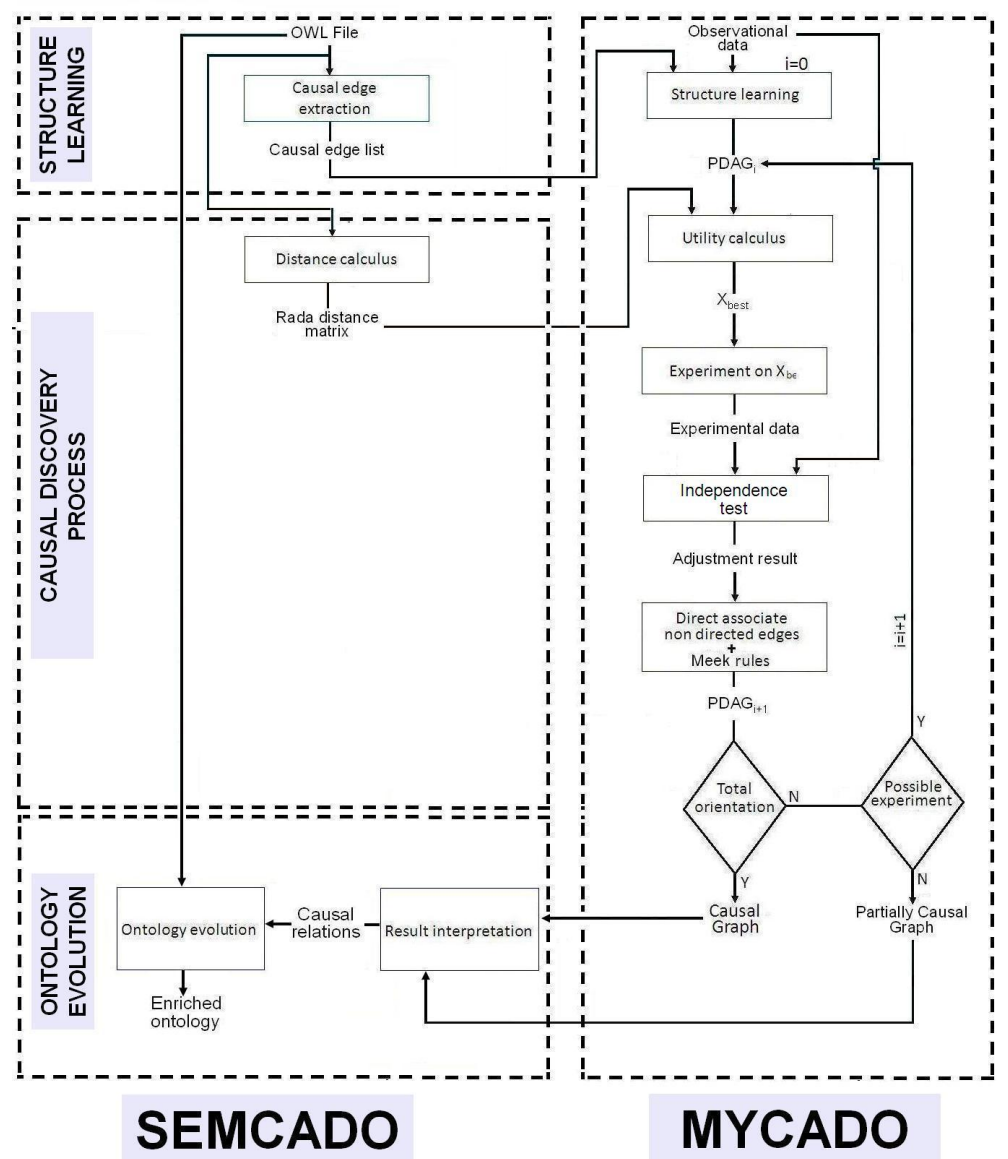

Fig. 1. SemCaDo: Extending MyCaDo to allow CBN-Ontology interactions

1) Learning the initial structure using causal prior knowledge: The ontology in input may contain some causal relations in addition to hierarchical and semantic relations. Those causal relations should be integrated from the beginning in the structure learning process in order to reduce the task complexity and better the final output. Therefore, such direct cause to effect relations will be incorporated as constraints when using structure learning algorithms. Our main objective is to narrow the corresponding search space by introducing some restrictions that all elements in this space must satisfy.

In our context, the only constraint that will be defined is edge existence. But we could also imagine in future work that some axioms in the ontology also give us some information about forbidden edges. All these edge constraints can easily be incorporated in usual BN structure learning algorithm [3]. Under some condition of consistency, these existence restrictions shall be fulfilled, in the sense that they are assumed to be true for the CBN representing the domain knowledge, and therefore all potential Partially Directed Acyclic Graph (PDAG) must 
necessarily satisfy them.

Definition 1. Given a domain ontology $\mathscr{O}$, let $G=\left(C, R_{c}\right)$ be the $D A G$ where $R_{c}: C_{i} \times C_{j}$ represents the subset of semantic causal relations extracted from $\mathscr{O}$. This subset included both direct and logically derivable semantic causal relations. Let $H=\left(X, E_{h}\right)$ be a PDAG, where $X$ is the set of the corresponding random variables and $E_{h}$ corresponds to the causal dependencies between them. $H$ is consistent with the existence restrictions in $G$ if and only if:

$\forall C_{i}, C_{j} \in C$, if $C_{i} \rightarrow C_{j} \in R_{c}$ then $X_{i} \rightarrow X_{j} \in E_{h}$.

When we are specifying the set of existence restrictions to be used, it is necessary to make sure that these restrictions can indeed be satisfied. In fact, such causal integration may lead to possible conflicts between the two models. When this occurs, we have to maintain the initial causal information in the PDAG since we are supposed to use perfect observational data. On the other hand, we should ensure the consistency of the existence restrictions in such a way that no directed cycles are created in G.

2) Causal discovery process: Before delving into the details of our approach, we first review the principal idea of the causal discovery process in MyCaDo algorithm [12].

When performing an experimentation on $X_{i}$, MyCaDo measure all neighboring variables and accordingly to the result direct all edges connecting $X_{i}$ and $\operatorname{Nei}\left(X_{i}\right)$. This edge orientation represents one instantiation $\left(\operatorname{inst}\left(A_{X_{i}}\right)\right)$ among all possible instantiations. It is then possible to continue the edge orientation by using the Meek rules [11] to infer new causal relations.

Let inferred $\left(\operatorname{inst}\left(A_{X_{i}}\right)\right)$ be the number of inferred edges based on $\operatorname{inst}\left(A_{X_{i}}\right)$. MyCaDo proposes that the utility of an experiment is related to the number of edges directly oriented or inferred, weighted by the cost of experiment $\left(\operatorname{cost}\left(A_{X_{i}}\right)\right)$ and measurement $\left(\operatorname{cost}\left(M_{X_{i}}\right)\right)$ :

$$
U\left(X_{i}\right)=\frac{\operatorname{Card}\left(N e i\left(X_{i}\right)\right)+C \operatorname{ard}\left(\text { inferred }\left(\operatorname{inst}\left(A_{X_{i}}\right)\right)\right)}{\alpha \operatorname{cost}\left(A_{X_{i}}\right)+\beta \operatorname{cost}\left(M_{X_{i}}\right)}
$$

where measures of importance $\alpha$ and $\beta \in[0,1]$ and $\operatorname{Card}(\mathrm{M})$ represents the cardinality of any set $\mathrm{M}$.

It seems obvious that the gained information of such utility function is essentially the node connectivity ( i.e. the number of undirected edges and those susceptible to be inferred) which serves to orient the maximal number of edges but not necessary the most informative ones.

To cope with this limitation, the strategy we propose in our approach makes use of a semantic distance calculus (e.g. Rada distance [16]) provided by the ontology structure. So, for each node in the graph, SemCaDo gives a generalization of the node connectivity by introducing the semantic inertia, denoted by $\operatorname{SemIn}\left(X_{i}\right)$ and expressed as follows: 


$$
\operatorname{SemIn}\left(X_{i}\right)=\frac{\sum_{X_{j} \in \operatorname{Nei}\left(X_{i}\right) \cup X_{i}} \operatorname{dist}_{\text {Rada }}\left(\operatorname{mscs}\left(\operatorname{Nei}\left(X_{i}\right) \cup X_{i}\right), X_{j}\right)}{\operatorname{Card}\left(\operatorname{Nei}\left(X_{i}\right) \cup X_{i}\right)}
$$

where:

- $\operatorname{mscs}\left(C_{i}, C_{j}\right)$ : the most specific common subsumer of the two concepts $C_{i}$ and $C_{j}$, where $i \neq j$.

- $\operatorname{dist}_{\text {Rada }}\left(C_{i}, C_{j}\right)$ : the shortest path between $C_{i}$ and $C_{j}$, where $i \neq j$,

Moreover, the semantic inertia presents three major properties:

- When the experimented variable and all its neighbors lie at the same level in the concept hierarchy, the semantic inertia will be equal to the number of hierarchical levels needed to reach the mscs.

- If the corresponding concepts belong to the same super-class then SemIn will be proportional to $\operatorname{Card}(\mathrm{Nei}()$.$) .$

- It essentially depends on semantic distance between the studied concepts. This means that the more this distance is important, the more the SemIn will be maximized.

By this way, we will accentuate the serendipitous aspect of the proposed strategy and investigate new and unexpected causal relations on the graph.

Further to these, we also integrate a semantic cumulus relative to the inferred edges denoted by Inferred_Gain in our utility function. For this purpose, we use $\mathrm{I}\left(X_{i}\right)$ to denote the set of nodes attached by inferred edges after performing an experimentation on $X_{i}$. So, the Inferred_Gain formula is expressed as follows:

$$
\operatorname{Inferred\_ Gain}\left(X_{i}\right)=\frac{\sum_{X_{j} \in I\left(X_{i}\right)} \operatorname{dist}_{\text {Rada }}\left(\operatorname{mscs}\left(I\left(X_{i}\right)\right), X_{j}\right)}{\operatorname{Card}\left(I\left(X_{i}\right)\right)}
$$

Inferred_Gain also represents a generalization of Card(inferred(inst(.)) and depends on the semantic distance between the studied concepts. Note that we don't use here all the information provided by the ontology. We should also consider the axioms to check if any new relation could be inferred from the semantic point of view. Better interacting with the axioms is one of our perspectives for future work.

When using the two proposed terms, our utility function will be as follows:

$$
U\left(X_{i}\right)=\frac{\operatorname{SemIn}\left(X_{i}\right)+\text { Inferred_Gain }\left(X_{i}\right)}{\alpha \operatorname{cost}\left(A_{X_{i}}\right)+\beta \operatorname{cost}\left(M_{X_{i}}\right)}
$$

where measures of importance $\alpha, \beta \in[0,1]$. 
This utility function will be of great importance to highlight the serendipitous character of SemCaDo algorithm by guiding the causal discovery process to investigate unexplored areas and conduct more informative experiments.

3) Edge orientation \& ontology evolution: Once the specified intervention performed, we follow the same edge orientation strategy as in MyCaDo [12]. So if there are still some non-directed edges in the PDAG, we re-iterate over the second phase and so on, until no more causal discoveries can be made. Since certain experimentation can not be performed, either because of ethical reasons or simply because it is impossible to do it, the final causal graph can be either a CBN or a partially causal graph.

In both cases, the causal knowledge will be extracted and interpreted for an eventual ontology evolution. In this way, the causal relations will be traduced as semantic causal relations between the corresponding ontology concepts.

We note that, because of the priority given to the ontology axioms, only causal relations guaranteeing the consistency will be retained for the ontology evolution process.

\subsection{Toy example}

In the following example, we briefly illustrate the various steps we followed to construct a $\mathrm{CBN}$ when using SemCaDo algorithm. As noted above, we assume that all random variables under study are modeled in the corresponding ontology (See Figure 2) as distinctly blue-colored concepts. We note that in order to simplify the semantic analysis, we restricted the ontology to only taxonomic relations and causal ones.

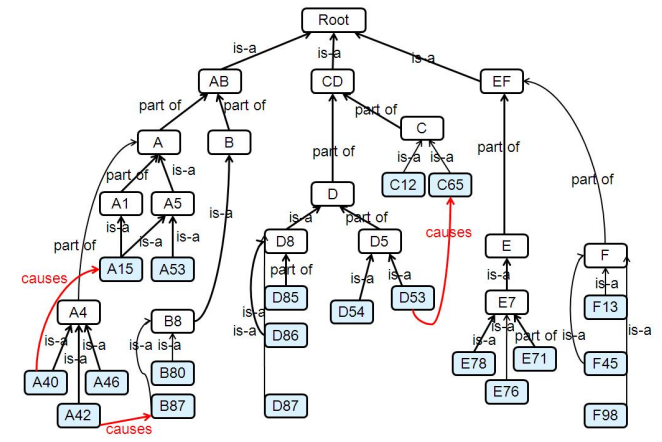

Fig. 2. An example of ontology: blue nodes denote the concepts under study, red relationships denote causal prior knowledge and black relationships are taxonomic relations.

As first step, we use the graph in Figure 3 (a) to specify the existence restrictions to be satisfied. In our case, three causal relations (i.e. A42 $\rightarrow$ B87, A40 $\rightarrow$ A15 and D53 $\rightarrow$ C65) extracted from the ontology in Figure 2 have to be modeled as directed arrows before learning the initial structure. Using this prior knowledge, 
Figure 3 (b) shows the resulting partially directed graph after performing a structure learning algorithm and applying the Meek rules [11] to infer edges.

When running the SemCaDo causal discovery process, the first best node to experiment on will be E71. This choice is strongly supported by the high semantic distance between E71 and its neighboring variables (i.e. E76 and A42) as well as the additional semantic cumulus relative to the edges susceptible to be inferred (i.e. E76-A40, A42-E76, A40-A42 and A40-E78). Thanks to this experimentation, we can investigate different causal relations between the more distant concepts in the ontology, in which we have not enough prior causal information (See Figure 2).

After finishing all the causal discovery step and learning the completed directed graph as shown in Figure 3 (c), the supplementary causal knowledge will be interpreted and reused in the corresponding ontology. The dashed lines in Figure 3 (d) indicate the ontology evolution (i.e. ontology enrichment) in response to SemCaDo discoveries.
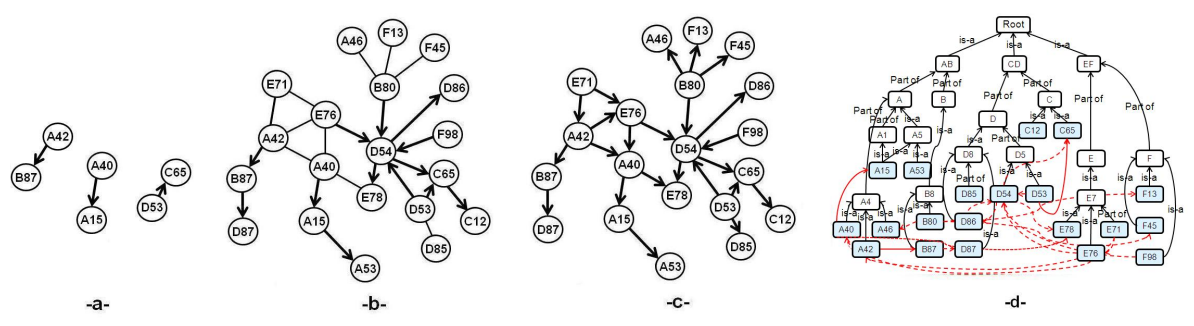

Fig. 3. (a) Existence restriction graph extracted from the ontology in Figure 2, (b) PDAG obtained after structure learning phase using previous restriction graph, (c) the CBN obtained after performing experiments on (E71, B80 and A42) and (d) the enriched ontology.

\section{Experimental study}

In the experimental evaluation, we will compare SemCaDo to MyCaDo algorithm [12] since both of them share the same assumptions and use the same input data.

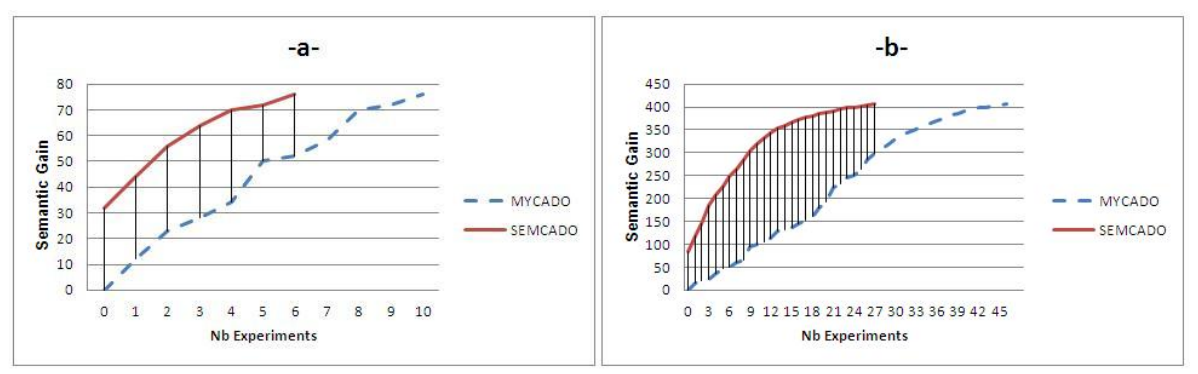

Fig. 4. The semantic gain given the number of experiments using MyCaDo and SemCaDo on relatively small graphs (a) and bigger ones (b) 
For this purpose, we randomly create a set of syntectic 50 and 200 node graphs and apply a DAG-to-CPDAG algorithm [2] on those CBNs in order to simulate the result of a structure learning algorithm working with a perfect infinite dataset. For each simulated graph, we automatically generate a corresponding concept hierarchy in which we integrate a varying percentage $(10 \%$ to $40 \%$ ) of the initial causal relations. As we do not dispose of a real system to intervene upon, we decide to simulate the experimentations directly in the previously generated CBNs as in [12].

Another point to consider in our experimental study concerns the calculation of the semantic gain. In fact, after each SemCaDo (resp. MyCaDo) iteration, we measure the sum of semantic distances (Rada's [16] in these experiments) relative to the new directed edges in the graph and update a semantic cumulus. In both strategies, the two corresponding curves are increasing in, meaning that the higher is the number of experimented variables, the higher is the value of the semantic gain. Nevertheless the more the curve is increasing faster, the more the approach is converging to the best and most impressive experiments.

Figure 4 shows that, during the experimentation process, our approach comfortably outperforms the MyCaDo algorithm in term of semantic gain. This is essentially due to the initial causal knowledge integration and the causal discovery strategy when performing the experimentations. But if the two curves reach the same maxima when obtaining a fully directed graph, where is the evolutionary contribution of SemCaDo? Let us remember that we are approaching a decision problem which is subject to the experimentation costs and the budget allocation. Taking into account this constraint, the domination of SemCaDo will be extremely beneficial when the number of experiments is limited.

All these experimental results show how the SemCaDo algorithm can adopt a serendipitous attitude with the minimum expected cost and effort. This is indeed a new avenue of causal investigation, moving far away from traditional techniques.

\section{Conclusions and Future Works}

In this paper, we outlined our serendipitous and cyclic approach which aims to i) integrate the causal prior knowledge contained in the corresponding ontology when learning the initial structure from observational data, ii) use a semantic distance calculus to guide the iterative causal discovery process to the more surprising relationships and iii) capture the required causal discoveries to be applied to ontology evolution. The SemCaDo algorithm is an initial attempt towards a more ambitious framework exploiting the power of BNs and ontologies. Future works will be devoted to ontology revolution through better interactions with the axioms during the causal discovery process.

\section{References}

1. M. Ben Messaoud, P. Leray, and N. Ben Amor. Integrating ontological knowledge for iterative causal discovery and visualization. In ECSQARU'09, pages 168-179, 
2009.

2. D. M. Chickering. Learning equivalence classes of bayesian-network structures. Journal of Machine Learning Research, 2:445-498, 2002.

3. L. M. de Campos and J. G. Castellano. Bayesian network learning algorithms using structural restrictions. International Journal of Approximate Reasoning, pages 233-254, 2007.

4. A. Devitt, B. Danev, and K. Matusikova. Constructing bayesian networks automatically using ontologies. In Second Workshop on Formal Ontologies Meet Industry. FOMI '06, Trento, Italy, 2006.

5. Z. Ding and Y. Peng. A probabilistic extension to ontology language OWL. In Proceedings of the 37th Hawaii International Conference on System Sciences (HICSS '04), 2004.

6. G. Flouris, D. Manakanatas, H. Kondylakis, D. Plexousakis, and G. Antoniou. Ontology change: classification and survey. In The Knowledge Engineering Review, volume 23, pages 117-152, 2008.

7. T. R. Gruber. Towards Principles for the Design of Ontologies Used for Knowledge Sharing. International Journal Human-Computer Studies Vol. 43, Issues 5-6, pages 907-928, November, 1995.

8. Y. B. He and Z. Geng. Active learning of causal networks with intervention experiments and optimal designs. JMLR, 9, pages 2523-2547, 2008.

9. B.-J. Jeon and I.-Y. Ko. Ontology-based semi-automatic construction of bayesian network models for diagnosing diseases in e-health applications. In FBIT, pages 595-602. IEEE Computer Society, 2007.

10. A. M. Khattak, K. Latif, S. Lee, and Y.-K. Lee. Ontology Evolution: A Survey and Future Challenges. In D. Ślzak, T.-h. Kim, J. Ma, W.-C. Fang, F. E. Sandnes, B.-H. Kang, and B. Gu, editors, U- and E-Service, Science and Technology, volume 62, pages 68-75. Springer Berlin Heidelberg, 2009.

11. C. Meek. Causal inference and causal explanation with background knowledge. In Proceedings of the Proceedings of the Eleventh Conference Annual Conference on Uncertainty in Artificial Intelligence (UAI-95), pages 403-41, San Francisco, CA, 1995. Morgan Kaufmann.

12. S. Meganck, P. Leray, and B. Manderick. Learning causal bayesian networks from observations and experiments: A decision theoretic approach. In V. Torra, Y. Narukawa, A. Valls, and J. Domingo-Ferrer, editors, MDAI, volume 3885 of Lecture Notes in Computer Science, pages 58-69. Springer, 2006.

13. K. P. Murphy. Active learning of causal bayes net structure. Technical report, University of California, Berkeley, USA, 2001.

14. J. Pearl. Probabilistic Reasoning in Intelligent Systems: Networks of Plausible Inference. Morgan Kaufmann Publishers Inc., San Francisco, CA, USA, 1988.

15. J. Pearl. Causality: models, reasoning, and inference. Cambridge University Press, 2000.

16. R. Rada, H. Mili, E. Bicknell, and M. Blettner. Development and application of a metric on semantic nets. IEEE Transactions on Systems, Man and Cybernetics, 19(1):17-30, 1989.

17. D. N. Xuan, L. Bellatreche, and G. Pierra. A versioning management model for ontology-based data warehouses. In A. M. Tjoa and J. Trujillo, editors, DaWaK, volume 4081 of Lecture Notes in Computer Science, pages 195-206. Springer, 2006.

18. Y. Yang and J. Calmet. Ontobayes: An ontology-driven uncertainty model. International Conference on Computational Intelligence for Modelling, Control and Automation (CIMCA), pages 457-463, 2005. 